\section{A Planar Microstrip Coupler with Enhanced Power Coupling}

\section{B.-K. Tan and G. Yassin}

In this Letter, we present the design of a planar microstrip coupler where power coupling between two parallel microstrip lines can easily be controlled. Enhancement of power coupling is done via two small slots in the ground plane underneath the microstrip pair. The ground plane slots force the field lines near the edge of each microstrip to cross to the adjacent microstrip. The magnitude of the power coupling can easily be controlled by adjusting the length of the slots. Here, we describe the operation of a microstrip coupler, and present the design of a $1 \times 4$ power distributor array that can be used to distribute the input microwave power uniformly to four branch lines arranged in a row. We also present the measured responses of the array, and show that the performance agree very well with simulated results.

Introduction: Direct coupling of power between two adjacent microstrip lines is beneficial in commonly used planar circuits because of the simplicity in fabrication and its compactness. This becomes crucially important at millimetre and sub-millimetre wavelengths where fabrication of transmission lines is done using complex photolithographic techniques. Common applications include their use as a directional coupler [1], an edge-coupled bandpass filter [2] and as a power distribution network that feeds several ports.

Traditional microstrip couplers use the fringing effect at the edge of the microstrip to couple power from one microstrip to another $[3,4]$. This method is often not effective, in particular in low impedance lines where the field lines are tightly confined between the microstrip layer and the ground plane, hence the coupled level of power is too low even for the smallest spacing permitted by modern photo-lithography. This is illustrated graphically at the top-right inset of Fig. 1. In this Letter, we present a novel method to enhance the power coupling without further narrowing down the gap between the two microstrip lines, by introducing two small slots at the ground plane underneath the two adjacent microstrips, as shown in Fig. 2. These slots force some field lines from each strip (that would otherwise have ended on the
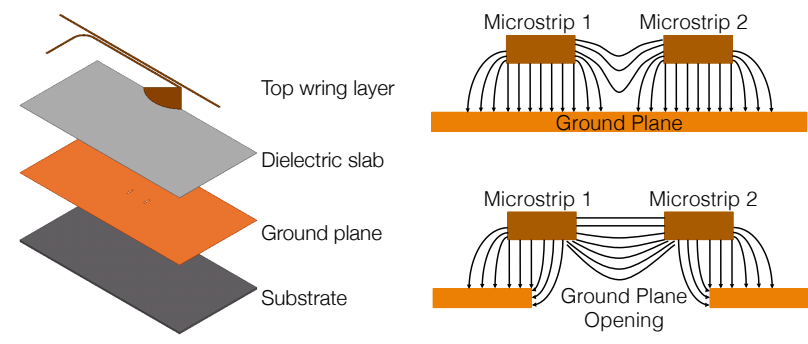

Fig. 1 The microstrip coupler unit assembly. Two small slots are cut in the ground plane to assist field lines cross-over.

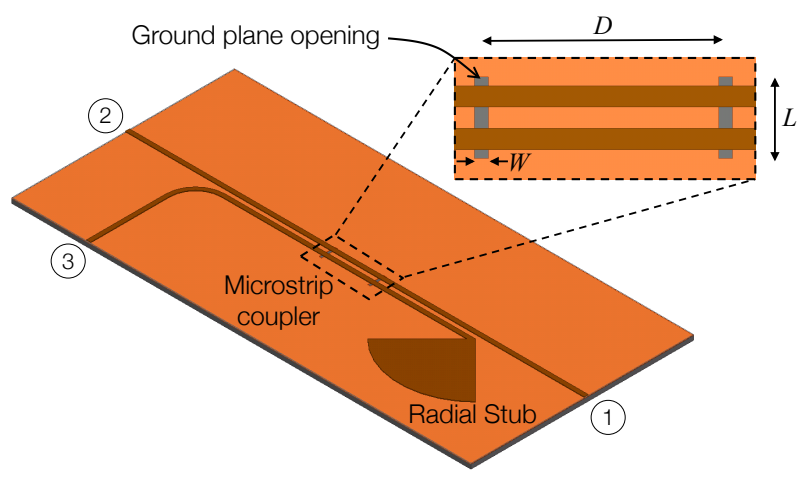

Fig. 2 Layout of a single unit microstrip coupler. The input port is denoted as Port 1, and the output port is denoted as Port 2, while the coupler branch is denoted as Port 3. The fourth port is terminated with a broadband open-circuit radial stub.

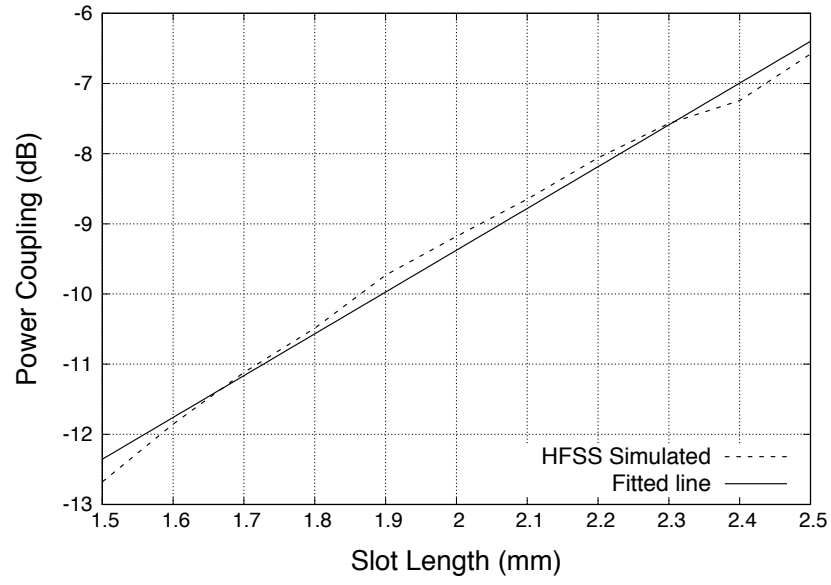

Fig. 3 The level of power coupled to the adjacent microstrip increases almost exponentially with the slot length $L$. The width and the distance between the two slots remain constant in this example.

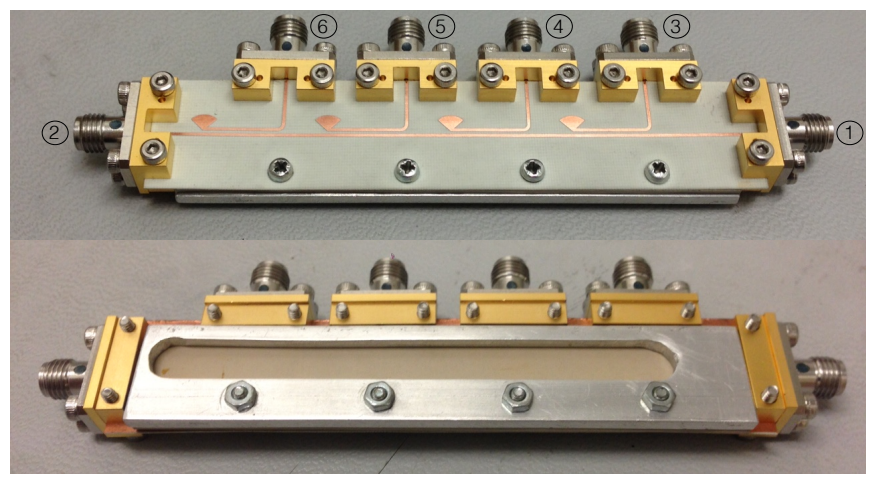

Fig. 4 Photo showing the $1 \times 4$ coupler array fabricated on a Roger RO 4350 printed circuit board, supported by a Roger Duroid 6010 bare substrate and an aluminium plate.

ground plane) to end on the other strip hence to be shared between the strips (see bottom-right inset of Fig. 1), therefore increasing the power coupling between the two microstrips. In principle, one slot is sufficient to improve the power coupling level, but the existence of a slot introduces unwanted inductance along the transmission line, changing the characteristic impedance of each microstrip. By using a pair of slots approximately quarter-wavelength apart, we are able to cancel out the extra inductance, hence maintain the characteristic impedance of the lines and consequently improve the return loss of the microstrip coupler.

Another important advantage of this design is that the coupling coefficient can be controlled by altering the length of the slots without changing the geometry of the coupler (the width and the location of the pair of slots only weakly affects the power couplings level). This is illustrated in Fig. 3 which shows that the level of power coupling between two microstrips varies exponentially with the length of the slots. This feature is very useful for designs that require flexibility in controlling the power coupling, for example in power distribution circuits that should split input power evenly between several output branches of an array.

Measurement and Results: Based on the method described above, we have designed a $1 \times 4$ microstrip coupler array that has constant $-10 \mathrm{~dB}$ power coupling from the input port to all four coupled ports, by cascading four microstrip couplers in series. The operational bandwidth is designated to be in the Ku-band, from $11-14 \mathrm{GHz}$ (approximately 24\% bandwidth). We used the electromagnetic simulator, Ansys High Frequency Structure Simulator (HFSS), to accurately model the microstrip coupler performance, especially to accurately compute the fringing effects. The drop of input power after each coupler is compensated for, by gradually increasing the slot length, keeping the power delivered to all coupled ports approximately constant at $-10 \pm$ $1 \mathrm{~dB}$ (approximately $\pm 2 \%$ variation at $-10 \mathrm{~dB}$ level). This illustrative design can be modified as required by specifications, for example to widen the bandwidth, or to reduce the power coupling variations. 


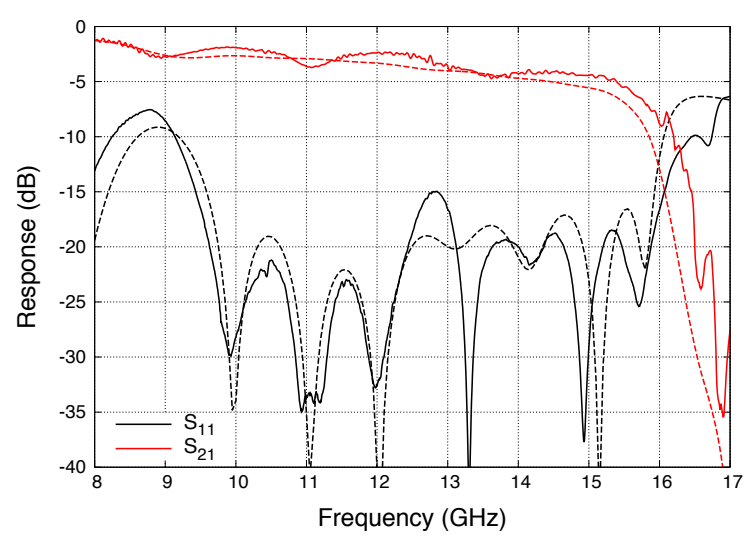

(a)

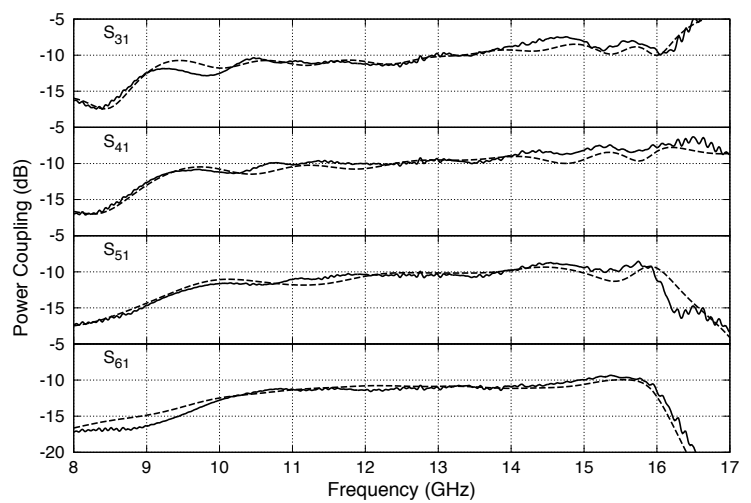

(b)

Fig. 5 (a) Transmission $\left(S_{21}\right)$ and return loss $\left(S_{11}\right)$ coefficients of the couplers array measured using the VNA (solid line) and calculated (dashed lined) using HFSS simulations. (b) Measured (solid line) and simulated (dashed line) coupling coefficients of each individual port from the input port $\left(S_{31}, S_{41}, S_{51}\right.$ and $\left.S_{61}\right)$. Notice the good agreement between the HFSS simulated results and the measured performances, especially within the designated frequency range of $11-14 \mathrm{GHz}$

The microstrip coupler array was printed on both sides of a $254 \mu \mathrm{m}$ thick Roger RO 4350 substrate $\left(\epsilon_{r}=3.66\right)$, and supported by a $1.27 \mathrm{~mm}$ thick Roger Duroid 6010 bare substrate $\left(\epsilon_{r}=10.2\right)$. A small aluminium plate with large clearance was bolted to the back of the assembly to strengthen the support for the device, as shown in Fig. 4. All 6 ports were connected to standard SMA connectors. The width of each microstrip and their separation were chosen at $0.5 \mathrm{~mm}$ which is convenient to fabricate using standard printed circuit technology at microwave frequencies and can also be easily fabricated when the dimensions are scaled to millimetre and sub-millimetre wavelengths using photo-lithography. In addition, the width of each microstrip yields a characteristic impedance of $50 \Omega$ to match the impedance of SMA connectors. The dimensions of the slots used in the tested device are tabulated in Table 1.

The transmission, coupling and return loss of the coupler array were measured using an Anritsu vector network analyser (VNA). The input signal was fed from the test port of the VNA which was connected to the Port 1 of the coupler chip (refer to Fig. 4 for the numbering of the ports). For measuring the scattering parameters from any of the coupled port (say Port 3), the output port of the VNA was connected to that port, with all the other remaining four ports terminated by $50 \Omega$ loads. This was then repeated until the responses of all 5 ports relative to the input port were measured. Fig. 5 (a) shows the measured results of the transmission and return loss performance, which agrees very well the HFSS simulated results. The measured resonance dips of the $S_{11}$ curve follow almost exactly the positions in the simulated return loss curve in the operation band, but at higher frequencies (above $15 \mathrm{GHz}$ ) the measured peaks are slightly lower than in simulations and the frequency of the resonances are slightly shifted. Nevertheless, the measured return loss remains well below $-15 \mathrm{~dB}$ from $10-15 \mathrm{GHz}$.

Fig. 5 (b) shows the measured power coupling from the input port to each individual coupled port. The measured couplings agree well with HFSS predictions, showing constant power coupling with a small
Table 1: Dimensions of the slots used in the $1 \times 4$ microstrip coupler array.

\begin{tabular}{|c|c|c|c|}
\hline Slot & Length $(L, \mathrm{~mm})$ & Width $(W, \mathrm{~mm})$ & Distance $(D, \mathrm{~mm})$ \\
\hline $1^{\text {st }}$ & 1.92 & 0.40 & 3.14 \\
$2^{\text {nd }}$ & 2.02 & 0.54 & 2.93 \\
$3^{\text {rd }}$ & 2.04 & 0.54 & 2.81 \\
$4^{\text {th }}$ & 2.17 & 0.47 & 2.79 \\
\hline
\end{tabular}

variation of $\pm 1 \mathrm{~dB}$ across a very large frequency range of $10-15.5 \mathrm{GHz}$, for all four coupled ports. The good agreement holds particularly well at the designated operating frequency band (11-14 GHz), with less than $0.5 \mathrm{~dB}$ discrepancy between the measured and predicted plots.

Conclusion: We have presented a microstrip coupler design that allows equal coupling of power from a single source to several ports using planar microstrip transmission lines at microwave frequencies. The design controls the coupling using a pair of slots in the ground plane. The performance is broadband and the coupler is easy to fabricate at both microwave and sub-millimetre wavelengths. For example, a design based on a $0.5 \mathrm{~mm}$ microstrip width and slot width at Ku-band is scaled to $\approx 7.5 \mu \mathrm{m}$ at $1 \mathrm{THz}$, which can easily be fabricated using standard photolithography. An important application of this design is the feeding of local oscillator (LO) power to a mixer detector array $[5,6]$ in modern radio-telescopes, to provide equal LO power to all detector pixels with the planar multiple port coupler circuit integrated to the detector array chip.

B. K. Tan and G. Yassin (Department of Physics (Astrophysics), University of Oxford, Denys Wilkinson Building, Keble Road, OX1 3 RH Oxford, United Kingdom.)

E-mail: boonkok.tan@physics.ox.ac.uk

\section{References}

1 Y. Chun, J. Moon, S. Yun, and J. Rhee, "Microstrip line directional couplers with high directivity," Electron. Lett, vol. 40, no. 5, pp. 317318, 2004.

2 Y. P. Zhang and M. Sun, "Dual-band microstrip bandpass filter using stepped-impedance resonators with new coupling schemes," IEEE Transactions on Microwave Theory and Techniques, vol. 54, no. 10, pp. 3779-3785, 2006

3 K. Gupta, R. Garg, I. Bahl, and P. Bhartia, Microstrip Lines and Slotlines. Arthech House, 1996.

4 D. M. Pozar, Microwave Engineering. John Wiley \& Sons, 2009.

5 J. Leech, G. Yassin, B.-. K. Tan, Y. J. Zhou, and P. Grimes, "An SIS mixer based focal-plane array at $230 \mathrm{GHz}$," in Twenty-six International Symposium on Space Terahertz Technology, 2015.

6 B.-K. Tan, G. Yassin, P. Grimes, and K. Jacobs, " 650 GHz SIS mixer fabricated on silicon-on-insulator substrate," Electronics Letters, vol. 49, no. 20, pp. 1273-1275, 2013. 